PROCEEDINGS OF THE

AMERICAN MATHEMATICAL SOCIETY

Volume 129, Number 7 , Pages $2017-2018$

S 0002-9939(00)05760-9

Article electronically published on November 30, 2000

\title{
NON-INVERTIBILITY OF CERTAIN ALMOST MATHIEU OPERATORS
}

\author{
R. BALASUBRAMANIAN, S. H. KULKARNI, AND R. RADHA
}

(Communicated by Joseph A. Ball)

\begin{abstract}
It is shown that the almost Mathieu operators of the type $T e_{n}=$ $e_{n-1}+\lambda \sin (2 n r) e_{n}+e_{n+1}$ where $\lambda$ is real and $r$ is a rational multiple of $\pi$ and $\left\{e_{n}: n=1,2,3, \ldots\right\}$, an orthonormal basis for a Hilbert space, is not invertible.
\end{abstract}

Let $H$ be a Hilbert space with an orthonormal basis $\left\{e_{n}: n=1,2,3, \ldots\right\}$. An important class of tridiagonal operators used in mathematical physics are almost Mathieu operators which are defined by

$$
T e_{n}=e_{n-1}+\lambda \cos (2 n \pi \alpha+\theta) e_{n}+e_{n+1},
$$

$\alpha, \lambda, \theta$ are real. Certain questions regarding the Lebesgue measure of the spectra of such operators seem to have received a good deal of attention in the literaure. (See 1], 2], 4].) However, the question of invertibility of such operators seems to be unexplored. In this note we prove that the almost Mathieu operators of the type

$$
T e_{n}=e_{n-1}+\lambda \sin (2 n r) e_{n}+e_{n+1},
$$

$\lambda$ real, $r$ a rational multiple of $\pi$ are not invertible. Since every separable Hilbert space is isometrically isomorphic to $\ell^{2}$, the main theorem is proved for operators on $\ell^{2}$.

Theorem 0.1. Let $V$ be an infinite tridiagonal matrix whose diagonal elements are $d_{1}, d_{2}, \ldots d_{m}, 0,-d_{m}, \ldots,-d_{1}, 0$ repeated in the same order and off diagonal entries are 1. Then $V$ defines a bounded linear operator on $\ell^{2}$ and $V$ is not invertible.

Proof. That $V$ defines a bounded linear operator on $\ell^{2}$ is straightforward. To show that $V$ is not invertible, we prove that $V$ is not onto. In particular we aim to show that $e_{1}$ is not in the range of $V$. Let $x=\left(\alpha_{1}, \alpha_{2}, \ldots\right) \in \ell^{2}$ such that $V x=(1,0,0, \ldots)$. Then $\alpha_{1} d_{1}+\alpha_{2}=1$ and

$$
\alpha_{n-1}+\alpha_{n} \lambda_{n}+\alpha_{n+1}=0, \quad n=1,2,3, \ldots,
$$

where $\lambda_{n}$ are the diagonal elements of the matrix, viz. $d_{1}, d_{2}, \ldots, d_{m}, 0,-d_{m}, \ldots$, $-d_{1}, 0$. We first consider a block of $2 m+3$ equations for $n=m+1$ to $3 m+3$. For $n=2 m+2, \lambda_{n}=0$, we have $\alpha_{2 m+3}=-\alpha_{2 m+1}$. Next we consider the two

Received by the editors June 18, 1999 and, in revised form, November 5, 1999.

2000 Mathematics Subject Classification. Primary 47B37; Secondary 15A15.

Key words and phrases. Almost Mathieu operator, determinant, tridiagonal matrix, tridiagonal operator. 
equations adjacent to the above for $n=2 m+1$ (with $\lambda_{n}=-d_{1}$ ) and $n=2 m+3$ (with $\lambda_{n}=d_{1}$ )

$$
\begin{gathered}
\alpha_{2 m}-d_{1} \alpha_{2 m+1}+\alpha_{2 m+2}=0, \\
\alpha_{2 m+2}+d_{1} \alpha_{2 m+3}+\alpha_{2 m+4}=0,
\end{gathered}
$$

This yields (using $\alpha_{2 m+1}=-\alpha_{2 m+3}$ ) $\alpha_{2 m+4}=\alpha_{2 m}$. Proceeding in this way we can prove by induction $\alpha_{2 m+2+k}=(-1)^{k} \alpha_{2 m+2-k}$ for $k=0,1,2, \ldots, m+1$. In particular $\alpha_{3 m+3}=(-1)^{m+1} \alpha_{m+1}$ and $\alpha_{3 m+2}=(-1)^{m} \alpha_{m+2}$. Now, the next block of $2 m+3$ equations for $n=3 m+3$ to $5 m+5$ is exactly same as the previous block. Hence as above, $\alpha_{5 m+5}=(-1)^{m+1} \alpha_{3 m+3}=\alpha_{m+1}$. Thus $\alpha_{n}= \pm \alpha_{m+1}$ for $n=m+1,3 m+3,5 m+5, \ldots$. Since $x \in \ell^{2}$, we have $\alpha_{m+1}=0$. Similarly $\alpha_{m+2}=0$. However, then $x=0$ and so $V x=e_{1}$ is impossible.

Now we consider a separable Hilbert sapce $H$ with an orthonormal basis $\left\{e_{n}\right.$ : $n=1,2,3, \ldots\}$ and the almost Mathieu operator of the type

$$
T e_{n}=e_{n-1}+\lambda \sin (2 n r) e_{n}+e_{n+1}
$$

where $\lambda$ is real, $r$ is rational multiple of $\pi$ say $\frac{p \pi}{q}$. Then using the properties of the sine function, we see that $T$ is a matrix of the type defined in Theorem 0.1 with a suitable choice of $m$. Thus we conclude the following result.

Corollary 0.2. Let $T e_{n}=e_{n-1}+\lambda \sin (2 n r) e_{n}+e_{n+1}, \lambda$ real and $r$ a rational multiple of $\pi$. Then $T$ is not invertible.

\section{ACKNOWLEDGEMENT}

The authors thank the refereee for several useful suggestions towards improving the presentation of this paper and in particular for suggesting an idea which led to a considerable simplification of the proof of Theorem 0.1 .

\section{REFERENCES}

1. J.Avron, P.H.M.V.Mouche and B.Simon, On the measure of the spectrum for the almost Mathieu equation, Com.Math. Phys. (132) 1990, 103-118. MR 92d:39014a

2. J.Bellisard, R.Lima and D.Testand, Cantor spectrum for the almost Mathieu equation, J.Funct. Anal. (48) 1982, 408-419. MR 84h:81019

3. S.Jitomirskaya and Y. Last, Anderson Localization, continuity of gaps and measure of the spectrum, Comm. Math. Phys. (195) 1998, 1-14. MR 99j:81038

4. Y.Last, Almost everything about the almost Mathieu operator, Proceedings of XI international congress of Math. Physics, Paris, 1994, Intl. Press (1995), 366-372. MR 96m:82034

5. Y.Last, Zero measure spectrum for the almoist Mathieu operator, Comm. Math. Phys. (164) 1994, 421-432. MR 95f:47096

The Institute of Mathematical Sciences, C.I.T. Campus, Madras-600 113, India

E-mail address: balu@imsc.ernet.in

Department of Mathematics, Indian Institute of Technology, Madras-600 036, India

E-mail address: shk@acer.iitm.ernet.in

Department of Mathematics, Anna University, Madras-600 025, India

E-mail address: radharam@annauniv.edu

E-mail address: radharam@imsc.ernet.in 